Gut and Liver, Vol. 9, No. 3, May 2015, pp. 395-404

\title{
Disease Progression in Chronic Hepatitis B Patients under Long-Term Antiviral Therapy
}

Jin Chang Moon, Seong Hun Kim, In Hee Kim, Chang Hun Lee, Sang Wook Kim, Seung Ok Lee, Soo Teik Lee, and DaeGhon Kim

Department of Internal Medicine, Research Institute of Clinical Medicine, Chonbuk National University Hospital, Chonbuk National University Medical School, Jeonju, Korea

See editorial on page 265.

Background/Aims: We investigated factors associated with the disease progression and development of hepatocellular carcinoma $(\mathrm{HCC})$ in chronic hepatitis $\mathrm{B}(\mathrm{CHB})$ patients during long-term oral nucleos(t)ide analog (NA) therapy. Methods: This retrospective study included 524 naive $\mathrm{CHB}$ patients who received oral NA therapy for more than 48 weeks between January 2003 and December 2007. The primary outcome was 5-year cumulative probability of disease progression and HCC development. Disease progression was defined as cirrhosis development, cirrhotic complications, HCC or liver-related mortality. Results: For the 524 patients, the cumulative probabilities of disease progression and HCC development at 1, 2, 3, 4 and 5 years were 1.1\%, 6.3\%, 9.0\%, $11.6 \%$, and $16.2 \%$ and $0.2 \%, 1.8 \%, 3.6 \%, 5.8 \%$, and $9.3 \%$, respectively. In multivariate analysis, age $>50$ years (hazard ratio $[H R], 1.05)$ and cirrhosis (HR, 2.95) were significant factors for disease progression. Similarly, age $>50$ years (HR, 1.05), family history of HCC (HR, 5.48), and cirrhosis (HR, 17.16) were significant factors for HCC development. Importantly, longer duration (>12 months) of maintained virological response $(<20 \mathrm{IU} / \mathrm{mL})$ reduced the risks of disease progression (HR, 0.19) and HCC development (HR, 0.09). Conclusions: Longer duration of maintained virological response significantly reduces the risk of disease progression or HCC development in CHB patients undergoing long-term oral NA therapy. (Gut Liver 2015;9:395-404)

Key Words: Antiviral therapy; Carcinoma, hepatocellular; Dis- ease progression; Hepatitis B, chronic

\section{INTRODUCTION}

Approximately 350 million people are estimated to be infected with hepatitis B virus (HBV). ${ }^{1}$ Chronic HBV infection is a main cause of liver cirrhosis, hepatic decompensation, and hepatocellular carcinoma (HCC). In the two recent Risk Evaluation of Viral Load Elevation and Associated Liver Disease/Cancer (REVEAL) studies from Taiwan, ${ }^{2,3}$ serum HBV DNA levels were independent predictors for progression to cirrhosis and HCC. The cumulative incidence of cirrhosis increased with the HBV DNA level ranging from $4.5 \%$ to $36.2 \%$ for patients with a hepatitis $B$ viral load of $<300$ copies $/ \mathrm{mL}$ and $>10^{6}$ copies/mL, respectively. ${ }^{3}$ The cumulative incidence of HCC increased proportionately with serum HBV DNA levels from 1.3\% in patients with HBV DNA levels $<300$ copies/mL to approximately $15 \%$ in patients with HBV DNA level $>10^{6}$ copies $/ \mathrm{mL}$. $^{2}$ Furthermore, all-cause and chronic liver disease mortality also increased with increasing HBV DNA levels. ${ }^{4}$

Oral nucleos(t)ide analogues (NAs) including lamivudine (LAM), telbivudine (LdT), clevudine (CLV), adefovir (ADV), entecavir (ETV), and tenofovir (TDF) have been successfully used to suppress serum HBV DNA levels. Previous studies reported that long-term LAM therapy reduced incidence of cirrhosis or HCC development compared to untreated control. ${ }^{5,6}$ However, long-term LAM therapy has been associated with a high rate of drug-resistant mutations leading to amelioration of antiviral therapy-related beneficial effects and disease progression. ${ }^{5-7}$ LdT and CLV show potent antiviral suppression in naive patients, but are associated with the substantial development of antiviral

Correspondence to: In Hee Kim

Department of Internal Medicine, Research Institute of Clinical Medicine, Chonbuk National University Hospital, Chonbuk National University Medical School, 20 Geonji-ro, Deokjin-gu, Jeonju 561-712, Korea

Tel: +82-63-250-1677, Fax: +82-63-254-1609, E-mail: ihkimmd@jbnu.ac.kr

Received on May 2, 2014. Revised on July 8, 2014. Accepted on July 9, 2014. Published online on December 5, 2014

Jin Chang Moon and Seong Hun Kim contributed equally to this work as first authors.

pISSN 1976-2283 eISSN 2005-1212 http://dx.doi.org/10.5009/gnl14170

() This is an Open Access article distributed under the terms of the Creative Commons Attribution Non-Commercial License (http://creativecommons.org/licenses/by-nc/3.0) which permits unrestricted non-commercial use, distribution, and reproduction in any medium, provided the original work is properly cited. 
resistance during long-term treatment. ${ }^{8,9} \mathrm{ADV}$ is effective in treatment-naive and LAM-resistant chronic hepatitis B (CHB) patients. ${ }^{10-12}$ The adverse effect of antiviral resistance during long-term LAM therapy can now be managed by the timely use of rescue therapy. ${ }^{13-15}$ Recently, more potent NAs including ETV or TDF can produce higher virologic response with very low risk of antiviral resistance. ${ }^{16-19}$ However, long-term data on the effect of these more potent NAs on the risk of developing cirrhosis and HCC are limited. It also remains debatable which factors are most important in affecting the risk of disease progression in CHB patients under long-term oral NA therapy.

In this study, we investigated the long-term effect of oral NA therapy on the disease progression and development of HCC in CHB patients. We also determined the factors associated with the disease progression and development of HCC during longterm NA therapy.

\section{MATERIALS AND METHODS}

\section{Patients}

This retrospective cohort study included naive CHB patients who had received oral NA therapy for at least 12 months between January 2003 and December 2012 at Chonbuk National University Hospital. Patients were eligible if they were 18 to 70 years of age and had CHB or HBV-related cirrhosis. CHB was defined as a detectable serum hepatitis B surface antigen (HBsAg) level for more than 6 months, serum HBV DNA level $\geq 2,000 \mathrm{IU} /$ $\mathrm{mL}$, and abnormal serum alanine aminotransferase (ALT) levels (>40 IU/L). Patients with a history of previous NAs treatment, HCC at baseline or diagnosed within 6 months after initiation of oral NA therapy, history of liver transplantation, coinfection with human immunodeficiency virus, autoimmune hepatitis, toxic hepatitis, and any other serious concurrent medical illness that might lead to an estimated life expectancy $<1$ year were excluded.

During the study period, 854 patients took oral NA therapy for at least 12 months at Chonbuk National University Hospital. We excluded 330 patients who had not met inclusion or exclusion criteria, i.e., 207 patients who had previous history of NA therapy, 76 patients with preexisting HCC at baseline or within the first 6 months of NA therapy, 14 patients with $<1$ year follow-up duration, two patients with the history of liver transplantation, and 31 patients with evidence of other malignant diseases. Accordingly, total 524 naive patients were included in the analysis. Since TDF had recently become available in Korea, oral antiviral therapy was initiated on one of the NAs including LAM, LdT, CLV or ETV (0.5 mg) after the diagnosis of CHB during the study period. ADV, ETV (1.0 mg), or, more recently, TDF were used as a rescue drug for patients with treatment failure, defined as emergence of virological breakthrough with or without genotypic resistance after initial NA therapy. On the judgment of the investigator, rescue therapy was also implemented for patients with suboptimal response, defined as persistent serum HBV DNA $\geq 2,000 \mathrm{IU} / \mathrm{mL}$ after initial LAM or LdT therapy for at least 6 months. This study was conducted in compliance with the World Medical Association Declaration of Helsinki and was approved by the Ethics Committee at our institution.

\section{Clinical data collection and serum assays}

Sociodemographic data, biochemical tests including liver function tests (ALT, albumin level, bilirubin level, and prothrombin time), renal tests, electrolytes (sodium, calcium, and phosphate levels), $\alpha$-fetoprotein, hepatitis B envelop antigen (HBeAg) and anti-HBe status, and HBV DNA level, type of initial oral NAs were collected at baseline. During the follow-up period, data collected at each visit included oral NA therapyrelated outcomes (serum ALT, HBeAg or anti-HBe status, serum HBV DNA, viral breakthrough, resistance, and changing antivirals), progression to cirrhosis from chronic hepatitis, any cirrhosis-related complications, HCC development, and death. Serum ALT was measured with an enzymatic assay and was defined as normal range < 40 IU/L. Serum HBsAg, and antibody to HBsAg and HBeAg were detected by an electrochemiluminescence immunoassay (Roche Diagnostics, Mannheim, Germany). Serum HBV DNA was quantified by real-time polymerase chain reaction (PCR) using the COBAS Taq-Man HBV quantitative test (Roche Molecular Systems, Branchburg, NJ, USA), which had a lower limit of quantification of $20 \mathrm{IU} / \mathrm{mL}$. In practice, serum HBV DNA was quantified every 3 months during NA therapy. Genotypic analysis of HBV DNA polymerase was performed in patients showing virological breakthrough during therapy using a matrix-assisted laser desorption/ionization time-of-flight mass spectrometry-based genotyping assay, previously known as the restriction fragment mass polymorphism assay. ${ }^{20}$

\section{Study endpoints and definitions}

The primary endpoint was the cumulative probability of disease progression during follow-up. Disease progression was defined as any of progression to cirrhosis from previous chronic hepatitis, cirrhosis-related complications, HCC, or liver-related death after initiation of oral NA therapy. The secondary endpoint was the cumulative probability of HCC development during followup. A diagnosis of cirrhosis was based on liver biopsies or radiologic findings with or without varices at upper gastrointestinal endoscopy. Radiologic findings of cirrhosis were defined as coarse liver echotexture with parenchymal nodularity, irregular surface nodularities, and the features of portal hypertension. ${ }^{21}$ Cirrhosis-related complications included ascites, variceal bleeding, hepatic encephalopathy, spontaneous bacterial peritonitis, and hepatorenal syndrome. Spontaneous bacterial peritonitis was defined as an ascitic fluid polymorphonuclear leukocyte count $\geq 250 / \mathrm{mm}^{3}$ with or without positive bacterial culture. ${ }^{21}$ The diagnosis of HCC was established based on typical radiologic features of HCC or histological confirmation as suggested 
by the European Association for the Study of the Liver guideline in 2012. ${ }^{22}$ Liver-related mortality was defined as death related to cirrhotic complications and/or HCC.

We also investigated the factors associated with disease progression and HCC development under oral NA therapy, which included baseline factors and on-treatment antiviral response. In relation to antiviral response, we evaluated virological response, duration of virological response, HBeAg seroconversion, virological breakthrough, and genotypic resistance. Virological response was defined as undetectable levels of serum HBV DNA determined by real-time PCR $(<20 \mathrm{IU} / \mathrm{mL}){ }^{23}$ Duration of maintained virological response was calculated by total duration of maintained undetectable serum HBV DNA levels by PCR $(<20$ $\mathrm{IU} / \mathrm{mL}$ ) under long-term NA therapy. Virological breakthrough was defined as a $\geq 1 \log _{10} \mathrm{IU} / \mathrm{mL}$ increase in serum HBV DNA levels from nadir in two consecutive samples 1 month apart while receiving NA treatment. ${ }^{23}$ Genotypic resistance was defined as the detection of HBV mutations known to confer antiviral resistance during antiviral therapy. ${ }^{23}$

\section{Statistical analyses}

Results are reported as mean \pm standard deviation. HBV DNA levels were logarithmically transformed for analysis. Continuous variables were compared using the two-tailed Student t-test. Categorical data were analyzed using the chi-square test or Fisher's exact test. Cumulative rates for disease progression and HCC development were evaluated by Kaplan-Meier analysis. Factors associated with disease progression and HCC development were analyzed by univariate and multivariate Cox regression models. A p-value $<0.05$ was considered statistically significant. Data were collected in Microsoft Excel 2007 (Microsoft Corp., Seattle, WA, USA) and analyzed using SPSS for Windows version 15.0 (SPSS Inc., Chicago, IL, USA).

\section{RESULTS}

\section{Baseline characteristics of study patients and flow of long-term NA therapy}

The baseline characteristics of the 524 naive patients are presented in Table 1 . The mean age was 46.2 years, 340 patients (64.9\%) were male, 360 patients (68.7\%) were HBeAg positive, and 194 patients (37.0\%) had cirrhosis. Of the 524 patients, Child-Pugh class A, B, and C comprised 450 (85.9\%), 64 (12.2\%), and $10(1.9 \%)$ patients, respectively. Serum HBV DNA level was $6.4 \pm 1.2 \log _{10} \mathrm{IU} / \mathrm{mL}$. The initial oral NAs were LAM, LdT, or CLV in 363 patients (69.3\%) and ETV in 161 patients (30.7\%). The median follow-up duration of patients was 48 months (range, 8 to 60 months). Of 363 patients initially receiving LAM, LdT or CLV, 112 patients (30.9\%) were continued each initial NA, but 251 patients (69.1\%) were changed to ADV (149 patients), ETV (90 patients), or other NAs (12 patients) during the study period. The reasons for change of initial NA in this group were genotypic resistance (193 patients), primary nonvirological response (eight patients), suboptimal response (12 patients), drug side effects (10 patients), and others (28 patients). Of the 161 patients in the initial ETV group, 157 (97.5\%) continued ETV therapy. Therapy was changed in four patients (2.5\%), and comprised ETV+ADV (two patients) and TDF (two patients) due to genotypic resistance (one patient), suboptimal response (two patients), and pregnancy (one patient).

\section{Cumulative incidence rates of disease progression and development of HCC}

Of the 524 patients, 67 showed disease progression during follow-up. The types of disease progression were progression to cirrhosis from previous chronic hepatitis (21 patients), development of cirrhosis-related complications (46 patients), HCC

Table 1. Baseline Characteristics of Study Patients $(n=524)$

\begin{tabular}{|c|c|}
\hline Characteristic & Value \\
\hline Age, yr & $46.2 \pm 12.2$ \\
\hline Male sex & $340(64.9)$ \\
\hline BMI, $\mathrm{kg} / \mathrm{m}^{2}$ & $23.9 \pm 3.1$ \\
\hline Diabetes & 59 (11.3) \\
\hline Alcohol consumption ( $\geq 20 \mathrm{~g} /$ day) & $135(25.8)$ \\
\hline Family history of HCC & $43 / 422(10.2)$ \\
\hline Coinfection with HCV & 4/467 (0.9) \\
\hline HBeAg positive & $360(68.7)$ \\
\hline Cirrhosis & $194(37.0)$ \\
\hline Platelets, $\times 10^{3} / \mathrm{mm}^{3}$ & $154.5 \pm 71.8$ \\
\hline PT (INR) & $1.1 \pm 0.2$ \\
\hline Serum AST, IU/L & $125.9 \pm 193.1$ \\
\hline Serum ALT, IU/L & $157.6 \pm 195.0$ \\
\hline Total bilirubin, mg/dL & $1.4 \pm 1.8$ \\
\hline Albumin, g/dL & $3.9 \pm 0.5$ \\
\hline Creatinine, mg/dL & $0.8 \pm 0.4$ \\
\hline Serum HBV DNA, $\log _{10} I U / m L$ & $6.4 \pm 1.2$ \\
\hline Child-Pugh score & $5.5 \pm 1.1$ \\
\hline Child-Pugh class $\mathrm{A} / \mathrm{B} / \mathrm{C}$ & $450(85.9) / 64(12.2) / 10(1.9)$ \\
\hline MELD score & $5.6 \pm 4.94$ \\
\hline \multicolumn{2}{|l|}{ Initial treatment NA } \\
\hline LAM & $293(55.9)$ \\
\hline CLV & $70(13.4)$ \\
\hline ETV & $161(30.7)$ \\
\hline Median follow-up duration (range), mo & $48(8-60)$ \\
\hline
\end{tabular}

Data are presented mean \pm SD or number (\%) unless otherwise indicated.

BMI, body mass index; HCC, hepatocellular carcinoma; HCV, hepatitis C virus; HBeAg, hepatitis B e antigen; PT (INR), prothrombin time (international normalized ratio); AST, aspartate aminotransferase; ALT, alanine aminotransferase; HBV, hepatitis B virus; MELD, model for end-stage liver disease; NA, nucleos(t)ide analog; LAM, lamivudine; CLV, clevudine; ETV, entecavir. 
A

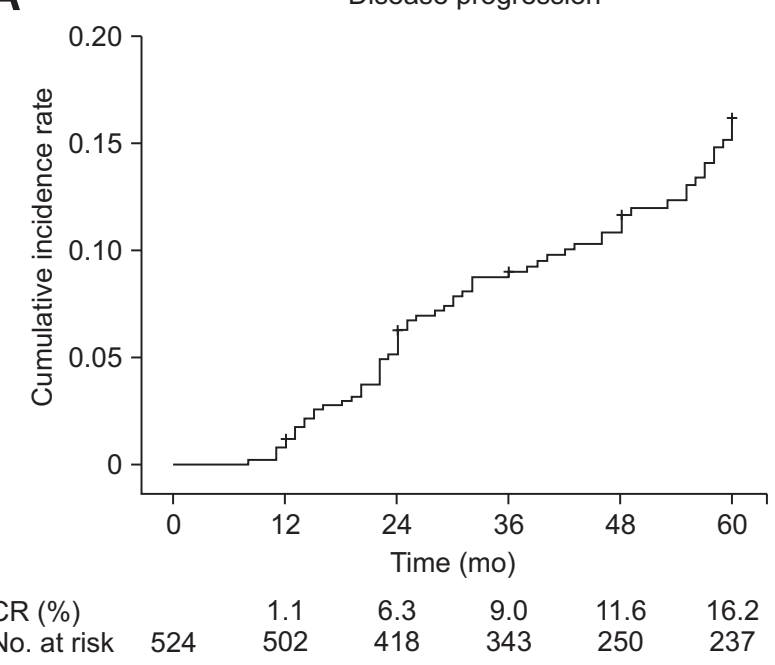

B

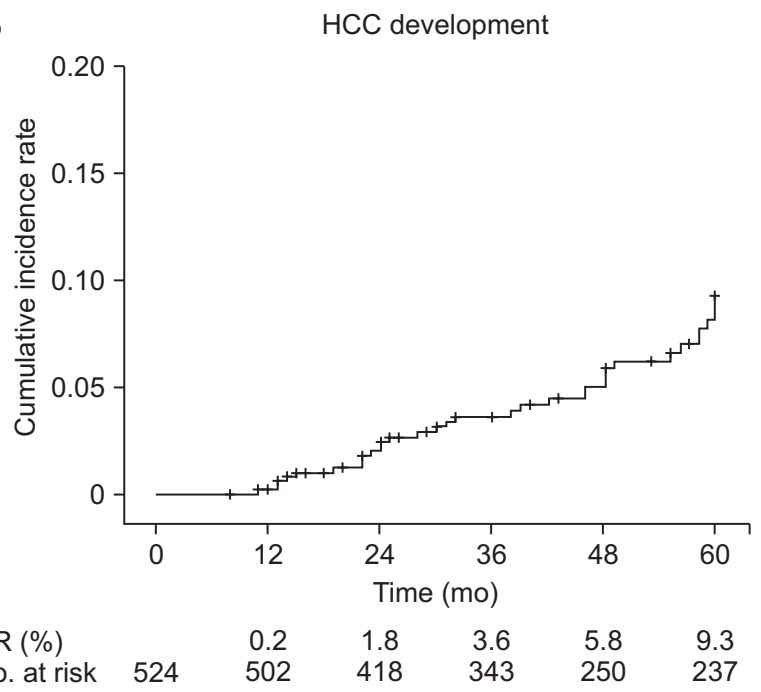

Fig. 1. Cumulative incidence rates of disease progression (A) and hepatocellular carcinoma (HCC) development (B) in all patients according to Kaplan-Meier analysis. Cumulative incidence rate of disease progression at 1, 2, 3, 4, and 5 years was 1.1\%, 6.3\%, 9.0\%, 11.6\%, and 16.2\%, respectively, and HCC development at 1, 2, 3, 4, and 5 years was $0.2 \%, 1.8 \%$, 3.6\%, 5.8\%, and 9.3\%, respectively.

CR, cumulative rate.

Table 2. Univariate Analysis of Factors Associated with Disease Progression and Hepatocellular Carcinoma Development

\begin{tabular}{|c|c|c|c|c|}
\hline \multirow{2}{*}{ Factor } & \multicolumn{2}{|c|}{ Disease progression } & \multicolumn{2}{|c|}{ HCC development } \\
\hline & Univariate HR (95\% CI) & p-value & Univariate HR (95\% CI) & p-value \\
\hline \multicolumn{5}{|l|}{ Baseline } \\
\hline Age, $>50$ yr & $3.37(2.04-5.55)$ & $<0.001$ & $5.42(2.53-11.61)$ & $<0.001$ \\
\hline Male sex & $1.09(0.65-1.83)$ & 0.732 & $1.59(0.72-3.51)$ & 0.252 \\
\hline BMI, $>25.0 \mathrm{~kg} / \mathrm{m}^{2}$ & $1.30(0.75-2.25)$ & 0.350 & $1.59(0.76-3.32)$ & 0.219 \\
\hline Diabetes & $3.19(1.84-5.53)$ & $<0.001$ & $4.07(1.94-8.52)$ & $<0.001$ \\
\hline Alcohol consumption ( $\geq 20 \mathrm{~g} /$ day) & $1.35(0.81-2.27)$ & 0.251 & $1.44(0.70-2.95)$ & 0.324 \\
\hline Family history of HCC & $1.96(0.99-3.85)$ & 0.052 & $3.47(1.56-7.74)$ & 0.002 \\
\hline Coinfection with HCV & $1.32(0.18-9.51)$ & 0.785 & $2.43(0.33-17.83)$ & 0.384 \\
\hline HBeAg positive & $0.71(0.43-1.17)$ & 0.178 & $0.41(0.21-0.81)$ & 0.009 \\
\hline Cirrhosis & $4.18(2.49-7.01)$ & $<0.001$ & $14.58(5.14-41.40)$ & $<0.001$ \\
\hline Platelets, $>10^{5} / \mathrm{mm}^{3}$ & $0.27(0.17-0.44)$ & $<0.001$ & $0.29(0.15-0.57)$ & $<0.001$ \\
\hline PT (INR), >1.2 & $2.61(1.61-4.24)$ & $<0.001$ & $2.79(1.42-5.50)$ & 0.003 \\
\hline Serum AST, IU/L > normal range & $1.68(0.53-5.34)$ & 0.382 & $1.26(0.30-5.25)$ & 0.752 \\
\hline Serum ALT, IU/L > normal range & $0.69(0.37-1.29)$ & 0.243 & $0.68(0.28-1.65)$ & 0.396 \\
\hline Total bilirubin, $>1.8 \mathrm{mg} / \mathrm{dL}$ & $2.06(1.17-3.61)$ & 0.012 & $1.16(0.46-3.07)$ & 0.726 \\
\hline Albumin, $>3.5 \mathrm{~g} / \mathrm{dL}$ & $0.48(0.29-0.80)$ & 0.004 & $0.45(0.22-0.91)$ & 0.027 \\
\hline Creatinine, $>1.4 \mathrm{mg} / \mathrm{dL}$ & $0.81(0.11-5.83)$ & 0.833 & $1.57(0.22-11.49)$ & 0.656 \\
\hline Child-Pugh class, $\geq \mathrm{B}$ & $1.91(1.06-3.44)$ & 0.032 & $1.62(0.67-3.93)$ & 0.282 \\
\hline MELD score, >7 & $2.36(1.46-3.81)$ & 0.001 & $2.65(1.35-5.19)$ & 0.005 \\
\hline \multicolumn{5}{|l|}{ On NA therapy } \\
\hline Virological response & $0.44(0.27-0.71)$ & 0.001 & $0.70(0.36-1.38)$ & 0.300 \\
\hline Duration of maintained virological response (>12 mo) & $0.29(0.17-0.47)$ & $<0.001$ & $0.41(0.21-0.80)$ & 0.009 \\
\hline Virologic breakthrough & $1.36(0.83-2.23)$ & 0.222 & $1.01(0.51-1.99)$ & 0.985 \\
\hline Genotypic resistance & $1.26(0.77-2.07)$ & 0.357 & $1.16(0.58-2.34)$ & 0.670 \\
\hline
\end{tabular}

HR, hazard ratio; CI, confidence interval; BMI, body mass index; HCC, hepatocellular carcinoma; HCV, hepatitis C virus; HBeAg, hepatitis B e antigen; PT (INR), prothrombin time (international normalized ratio); AST, aspartate aminotransferase; ALT, alanine aminotransferase; MELD, model for end-stage liver disease; NA, nucleos(t)ide analog. 
(34 patients), and liver-related death (five patients). Overall, the cumulative incidence rates of disease progression and HCC development in CHB patients under long-term oral NA therapy increased as the treatment duration increased. The cumulative incidence rates of disease progression were $1.1 \%$ at 1 year, $6.3 \%$ at 2 years, $9.0 \%$ at 3 years, $11.6 \%$ at 4 years, and $16.2 \%$ at 5 years (Fig. 1A). The cumulative incidence rates of HCC development were $0.2 \%$ at 1 year, $1.8 \%$ at 2 years, 3.6\% at 3 years, 5.8\% at 4 years, and $9.3 \%$ at 5 years (Fig. 1B).

\section{Factors associated with disease progression and HCC development}

Tables 2 and 3 display results of univariate and multivariate analyses of factors associated with disease progression and HCC development during follow-up. In the univariate analysis (Table 2), age $>50$ years, diabetes, underlying cirrhosis, platelet $\leq 10^{5} /$ $\mathrm{mm}^{3}$, prothrombin time (international normalized ratio) $>1.2$, total bilirubin $>1.8 \mathrm{mg} / \mathrm{dL}$, albumin $\leq 3.5 \mathrm{~g} / \mathrm{dL}$, Child-Pugh class $\geq B$, model for end-stage liver disease score $>7$ points, virological response, and duration of maintained virological response

Table 3. Multivariate Analysis of Factors Associated with Disease Progression and Hepatocellular Carcinoma Development by Cox Regression Model

\begin{tabular}{|c|c|c|c|c|}
\hline \multirow{2}{*}{ Factor } & \multicolumn{2}{|c|}{ Disease progression } & \multicolumn{2}{|c|}{ HCC development } \\
\hline & Adjusted HR $(95 \% \mathrm{CI})$ & $\mathrm{p}$-value & Adjusted HR (95\% CI) & p-value \\
\hline Age, $>50 \mathrm{yr}$ & $1.05(1.02-1.07)$ & $<0.001$ & $1.05(1.01-1.10)$ & 0.016 \\
\hline Family history of HCC & - & - & $5.48(2.13-14.09)$ & 0.004 \\
\hline Cirrhosis & $2.95(1.48-5.88)$ & 0.002 & $17.16(4.67-63.12)$ & $<0.001$ \\
\hline Duration of maintained virological response (>12 mo) & $0.19(0.09-0.37)$ & $<0.001$ & $0.09(0.04-0.21)$ & $<0.001$ \\
\hline
\end{tabular}

HCC, hepatocellular carcinoma; HR, hazard ratio; CI, confidence interval.

Table 4. Comparison of Baseline Characteristics and Treatment Response between the Progression to Cirrhosis Group and Stable Group among 330 Patients with Chronic Hepatitis B

\begin{tabular}{|c|c|c|c|}
\hline Factor & Progression to cirrhosis group $(\mathrm{n}=21)$ & Stable group (n=309) & p-value \\
\hline Age, yr & $50.3 \pm 9.9$ & $42.2 \pm 11.8$ & 0.002 \\
\hline Male sex & $13(61.9)$ & $208(67.3)$ & 0.610 \\
\hline BMI, $\mathrm{kg} / \mathrm{m}^{2}$ & $15.8 \pm 10.6$ & $15.1 \pm 10.0$ & 0.767 \\
\hline Diabetes & $5(23.8)$ & $18(5.8)$ & 0.010 \\
\hline Alcohol consumption ( $\geq 20 \mathrm{~g} /$ day) & $6(28.6)$ & $80(25.9)$ & 0.799 \\
\hline Family history of HCC & $1(5.9)$ & $25(10.2)$ & 1.000 \\
\hline Coinfection with HCV & 0 & $1(0.4)$ & 1.000 \\
\hline HBeAg positive & $18(85.7)$ & $226(73.1)$ & 0.304 \\
\hline Platelets, $\times 10^{3} / \mathrm{mm}^{3}$ & $131.1 \pm 39.2$ & $189.2 \pm 56.8$ & $<0.001$ \\
\hline PT (INR) & $1.2 \pm 0.3$ & $1.1 \pm 0.1$ & 0.056 \\
\hline Serum AST, IU/L & $144.2 \pm 112.8$ & $319.2 \pm 230.4$ & 0.920 \\
\hline Serum ALT, IU/L & $159.4 \pm 155.0$ & $192.1 \pm 221.1$ & 0.506 \\
\hline Total bilirubin, $\mathrm{mg} / \mathrm{dL}$ & $1.6 \pm 2.6$ & $1.1 \pm 1.5$ & 0.439 \\
\hline Serum HBV DNA, $\log _{10} \mathrm{IU} / \mathrm{mL}$ & $6.7 \pm 1.1$ & $6.6 \pm 1.3$ & 0.763 \\
\hline \multicolumn{4}{|l|}{ Initial treatment NA } \\
\hline LAM or CLV vs ETV & 17 (81.0) vs 4 (19.0) & 220 (71.2) vs 89 (28.8) & 0.336 \\
\hline Virological response & $11(52.4)$ & $236(76.4)$ & 0.140 \\
\hline Duration of maintained virological response ( $>12 \mathrm{mo}$ ) & $6(28.6)$ & $186(60.2)$ & 0.006 \\
\hline Virologic breakthrough & $9(42.9)$ & $158(51.1)$ & 0.463 \\
\hline Genotypic resistance & $8(42.1)$ & $127(44.3)$ & 0.855 \\
\hline
\end{tabular}

Data are presented mean \pm SD or number $(\%)$.

BMI, body mass index; HCC, hepatocellular carcinoma; HCV, hepatitis C virus; HBeAg, hepatitis B e antigen; PT (INR), prothrombin time (international normalized ratio); AST, aspartate aminotransferase; ALT, alanine aminotransferase; HBV, hepatitis B virus; NA, nucleos(t)ide analog; LAM, lamivudine; CLV, clevudine; ETV, entecavir. 
were significantly associated with disease progression. Similarly, age $>50$ years, diabetes, family history of HCC, HBeAg positivity, underlying cirrhosis, platelet $\leq 10^{5} / \mathrm{mm}^{3}$, prothrombin time (international normalized ratio) $>1.2$, albumin $\leq 3.5 \mathrm{~g} / \mathrm{dL}$, model for end-stage liver disease score $>7$ points, and duration of maintained virological response were significantly associated with HCC development. In the multivariate analysis (Table 3), age $>50$ years (hazard ratio [HR], 1.05; 95\% confidence interval [CI], 1.02 to 1.07 ) and cirrhosis (HR, 2.95; 95\% CI, 1.48 to 5.88) were significant factors for disease progression. Similarly, age $>50$ years ( $\mathrm{HR}, 1.05 ; 95 \% \mathrm{CI}, 1.01$ to 1.10 ), family history of HCC (HR, 5.48; 95\% CI, 2.13 to 14.09), and cirrhosis (HR, 17.16; 95\% CI, 4.67 to 63.12) were significant factors for HCC development. Furthermore, longer duration (>12 months) of maintained virological response lowered the risks of disease progression $(\mathrm{HR}$, 0.19; 95\% CI, 0.09 to 0.37) and HCC development (HR, 0.09; 95\% CI, 0.04 to 0.21) (Table 3).

In this study, 21 patients (6.4\%) among 330 patients with $\mathrm{CHB}$ progressed to cirrhosis during antiviral therapy. When we compared baseline characteristics and treatment response between the progression to cirrhosis group and stable CHB group, the progression to cirrhosis was associated with older age, diabetes, lower platelet count, shorter duration of maintained virological response (Table 4). We also analyzed the clinical characteristics of patients who achieved maintained virological response ( $>12$ months) among total study patients. HBeAg negativity, presence of cirrhosis, lower platelet count, higher serum AST level, lower baseline serum HBV DNA level, initial treatment with ETV were associated with achievement of maintained virological response (>12 months) (Table 5).

\section{Cumulative incidence of disease progression and HCC development in subgroup analysis}

In the subgroup analysis, the cumulative incidence rates of disease progression were significantly higher among patients with age $>50$ years versus $\leq 50$ years $(1.5 \%$ vs $0.9 \%$ at 1 year, $10.3 \%$ vs $3.8 \%$ at 2 years, $14.7 \%$ vs $5.5 \%$ at 3 years, $19.2 \%$ vs $7.2 \%$ at 4 years, and $29.3 \%$ vs $8.8 \%$ at 5 years; $p<0.001$ ) (Fig. 2A). The cumulative incidence rates of disease progression were also significantly higher among patients with pre-existing cirrhosis versus noncirrhosis $(2.1 \%$ vs $0.6 \%$ at 1 year, $11.8 \%$ vs $3.1 \%$ at 2 years, $15.5 \%$ vs $5.2 \%$ at 3 years, $22.7 \%$ vs $5.2 \%$ at 4 years, and $30.2 \%$ vs $8.0 \%$ at 5 years; $p<0.001$ ) (Fig. 2B). Furthermore, the cumulative incidence rates of disease progression were significantly lower among patients with longer duration (>12 months) of maintained virological response compared to those with shorter duration ( $\leq 12$ months) $(0.0 \%$ vs $3.1 \%$ at 1 year, $0.6 \%$ vs $16.6 \%$ at 2 years, $3.4 \%$ vs $19.1 \%$ at 3 years, $6.0 \%$ vs $21.9 \%$ at 4 years, and $10.7 \%$ vs $26.2 \%$ at 5 years; $p<0.001$ ) (Fig. 2C). The cumulative incidence rate of HCC development was significantly higher among patients with age $>50$ years versus $\leq 50$ years $(0.5 \%$ vs $0.0 \%$ at 1 year, $5.3 \%$ vs $0.6 \%$ at 2 years,

Table 5. Comparison of Clinical Characteristics of Patients with Maintained Virological Response (VR) (>12 Months) versus Those without Maintained VR

\begin{tabular}{lccc}
\hline \multicolumn{1}{c}{ Factor } & With maintained VR $(\mathrm{n}=330)$ & Without maintained VR $(\mathrm{n}=194)$ & $\mathrm{p}$-value \\
\hline Age, $\mathrm{yr}$ & $46.5 \pm 12.2$ & $45.7 \pm 12.2$ & 0.508 \\
Male sex & $210(63.6)$ & $130(67.0)$ & 0.435 \\
BMI, $\mathrm{kg} / \mathrm{m}^{2}$ & $15.8 \pm 10.0$ & $17.0 \pm 9.9$ & 0.207 \\
Diabetes & $35(10.6)$ & $24(12.4)$ & 0.537 \\
Alcohol consumption $(\geq 20 \mathrm{~g} /$ day) & $79(23.9)$ & $56(28.9)$ & 0.213 \\
Family history of HCC & $30(11.0)$ & $13(8.7)$ & 0.462 \\
Co-infection with HCV & $2(0.7)$ & $2(1.1)$ & 0.631 \\
HBeAg positive & $192(58.2)$ & $168(86.6)$ & $<0.001$ \\
Cirrhosis & $138(41.8)$ & $56(28.9)$ & 0.003 \\
Platelets, $10^{3} / \mathrm{mm}{ }^{3}$ & $149.3 \pm 70.8$ & $163.3 \pm 72.9$ & 0.031 \\
PT (INR) & $1.1 \pm 0.2$ & $1.1 \pm 0.2$ & 0.381 \\
Serum AST, IU/L & $137.6 \pm 230.3$ & $106.0 \pm 100.2$ & 0.031 \\
Serum ALT, IU/L & $165.3 \pm 220.1$ & $144.6 \pm 142.1$ & 0.193 \\
Total bilirubin, mg/dL & $1.5 \pm 1.9$ & $1.2 \pm 1.6$ & 0.110 \\
Serum HBV DNA, log ${ }_{10} \mathrm{IU} / \mathrm{mL}$ & $6.2 \pm 1.3$ & $6.7 \pm 1.2$ & $<0.001$ \\
Initial treatment NA & & $157(80.9)$ vs $37(19.1)$ & $<0.001$ \\
LAM or CLV vs ETV & $206(62.4)$ vs $124(37.6)$ & & $<$ \\
\hline
\end{tabular}

Data are presented mean \pm SD or number $(\%)$.

BMI, body mass index; HCC, hepatocellular carcinoma; HCV, hepatitis C virus; HBeAg, hepatitis B e antigen; PT(INR), prothrombin time (international normalized ratio); AST, aspartate aminotransferase; ALT, alanine aminotransferase; HBV, hepatitis B virus; NA, nucleos(t)ide analogue; LAM, lamivudine; CLV, clevudine; ETV, entecavir. 
A

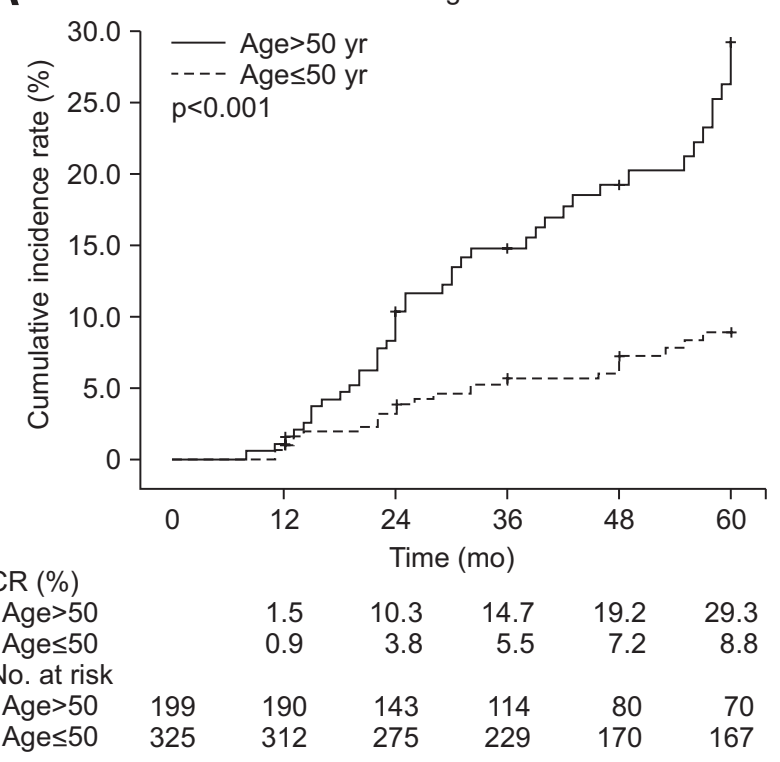

C

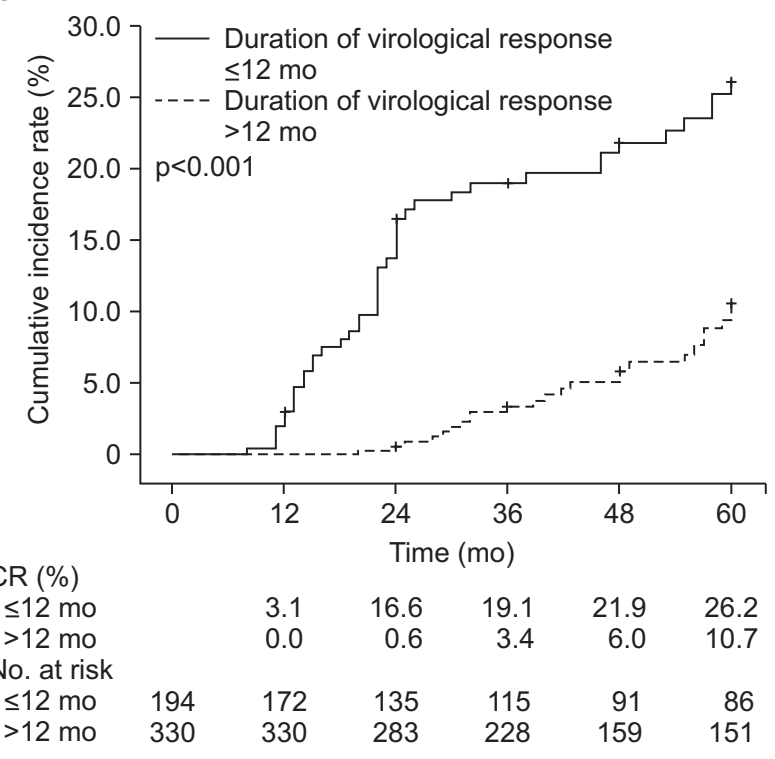

$7.3 \%$ vs $1.4 \%$ at 3 years, $10.6 \%$ vs $3.1 \%$ at 4 years, and $19.7 \%$ vs $3.7 \%$ at 5 years; $\mathrm{p}<0.001$ ) (Fig. $3 \mathrm{~A}$ ). The cumulative incidence rates of HCC development were also significantly higher among patients with pre-existing cirrhosis versus noncirrhosis $(0.5 \%$ vs $0.0 \%$ at 1 year, $6.1 \%$ vs $0.3 \%$ at 2 years, $9.4 \%$ vs $0.3 \%$ at 3 years, $15.6 \%$ vs $0.3 \%$ at 4 years, and $21.9 \%$ vs $2.1 \%$ at 5 years; $\mathrm{p}<0.001$ ) (Fig. 3B). The cumulative incidence rates of HCC development were also significantly higher among patients with family history of HCC versus without family history of HCC (2.3\% vs $0.0 \%$ at 1 year, $11.7 \%$ vs $2.0 \%$ at 2 years, $14.9 \%$ vs $3.2 \%$ at 3 years, $14.9 \%$ vs $6.4 \%$ at 4 years, and $26.2 \%$ vs $9.0 \%$ at 5 years; $\mathrm{p}=0.001$ ) (Fig. 3C). Furthermore, the cumulative incidence rates of HCC development were significantly lower among patients with longer duration (>12 months) of maintained virological
B Pre-existing cirrhosis

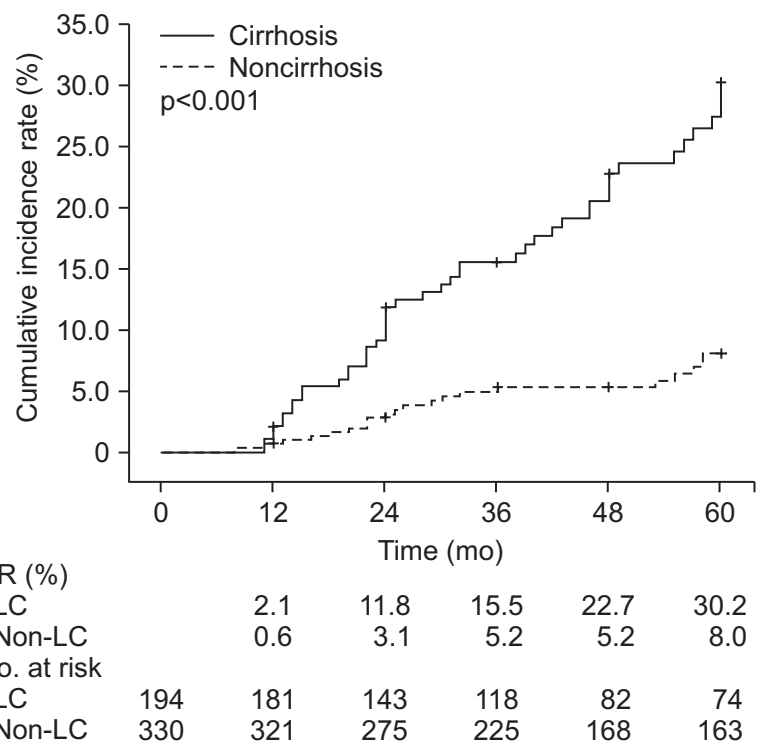

Fig. 2. Cumulative incidence rates of disease progression in subgroup analysis. (A) Age $>50$ years versus $\leq 50$ years, (B) pre-existing cirrhosis versus noncirrhosis, (C) duration of maintained virological response $\leq 12$ months versus $>12$ months. Log-rank p-value.

$\mathrm{CR}$, cumulative rate; $\mathrm{LC}$, liver cirrhosis.

response compared to those with shorter duration ( $\leq 12$ months) ( $0.0 \%$ vs $0.5 \%$ at 1 year, $0.3 \%$ vs $6.5 \%$ at 2 years, $2.1 \%$ vs $6.5 \%$ at 3 years, $3.8 \%$ vs $9.7 \%$ at 4 years, and $6.9 \%$ vs $13.7 \%$ at 5 years; $\mathrm{p}=0.007$ ) (Fig. 3D).

\section{DISCUSSION}

In this study, the cumulative incidence rates of disease progression and HCC development in CHB patients undergoing long-term oral NA therapy were increased according to the treatment duration from $1.1 \%$ and $0.2 \%$ at 1 year to $16.2 \%$ and 9.3\% at 5 years, respectively. Among baseline factors, age $>50$ years and pre-existing cirrhosis were significantly associated with disease progression and age $>50$ years, family history of 
A

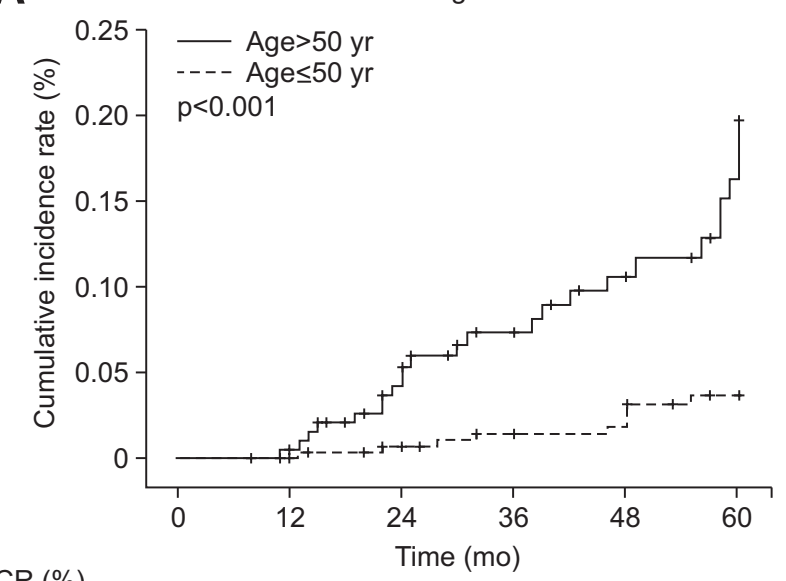

\section{C}

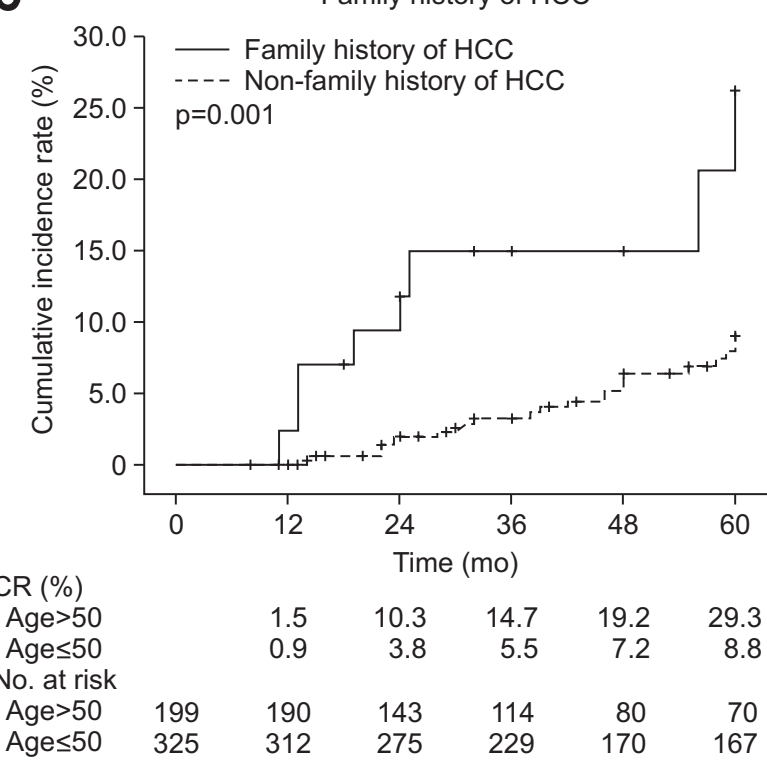

B

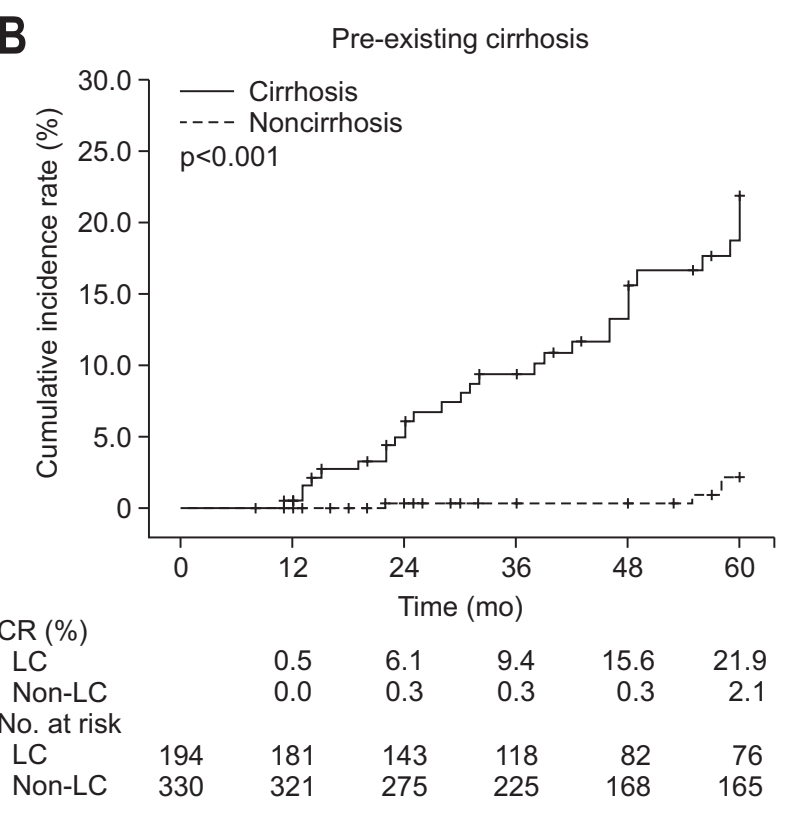

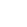

D

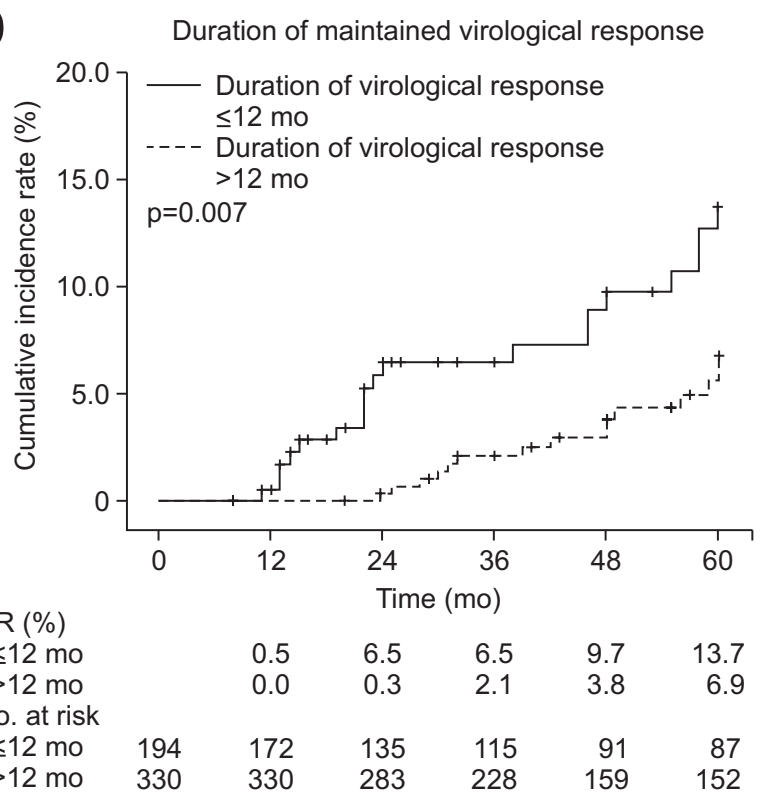

Fig. 3. Cumulative incidence rates of hepatocellular carcinoma (HCC) development in subgroup analysis. (A) Age $>50$ years versus $\leq 50$ years, (B) pre-existing cirrhosis versus noncirrhosis, (C) with or without family history of HCC, (D) duration of maintained virological response $\leq 12$ months versus $>12$ months.

$\mathrm{CR}$, cumulative rate.

HCC, and pre-existing cirrhosis were significantly associated with HCC development. Furthermore, longer duration ( $>12$ months) of maintained virological response significantly lowered the risk of disease progression and HCC development in CHB patients undergoing long-term oral NA therapy.

Long-term LAM therapy leads to reversal of advanced fibrosis $^{24,25}$ and lowers the cumulative rate of cirrhosis and/or HCC development as compared with untreated controls. ${ }^{5,6}$ A double-blind, randomized, controlled trial demonstrated that maintenance LAM therapy in $436 \mathrm{CHB}$ patients with cirrhosis or advanced fibrosis (Ishak fibrosis score $\geq 4$ ) significantly reduced overall disease progression $(7.8 \%$ vs $17.7 \%, p=0.001)$ and incidence of HCC (3.9\% vs 7.4\%, $\mathrm{p}=0.047$ ), as compared with untreated controls during median follow-up of 32.4 months. ${ }^{6}$ However, long-term LAM therapy has been associated with a high rate of drug-resistant mutations. ${ }^{5}$ These patients were more likely to experience disease progression and to die because of reasons related to the worsening of liver function. ${ }^{6}$ On the other hand, as the development of new oral NAs has proven effective in LAM-resistant mutants, the negligible effect of LAM resis- 
tance can be overcome by the timely use of rescue therapy. ${ }^{13-15}$ A Japanese study involving 158 patients with LAM-resistant $\mathrm{CHB}$ rescued by $\mathrm{ADV}$ add-on therapy showed that the rate of virological response was $90.8 \%$ after 4 years of treatment. ${ }^{26}$ Therefore, it is anticipated that long-term therapy with ADV rescue would have similar or even better long-term outcomes because of their lower rate of drug resistance and similar crossresistance profile. ETV and TDF were recently recommended as first-line drug for naive $\mathrm{CHB}$ patients due to more potent viral suppression with very low or no antiviral resistance. ${ }^{23}$ A large cohort study in Hong Kong demonstrated that an ETV treated cohort with liver cirrhosis had significantly lower cumulative probabilities of hepatic events (21.6\% vs $33.9 \%$ at 3 years and $25.5 \%$ vs $45.9 \%$ at 5 years), HCC (9.1\% vs $14.5 \%$ at 3 years and $13.8 \%$ vs $26.4 \%$ at 5 years) compared to the control cohort. ${ }^{27}$ Another study from Japan involving 472 ETV treated patients also reported significantly lower cumulative HCC incidence rates at 5 years in the ETV group than in the nontreated control group (3.7\% vs 13.7\%, p<0.001). ${ }^{28}$ Our study included patients who initiated oral NA therapy including LAM, LdT, CLV, and ETV. In the results, the overall 3-year and 5-year cumulative incidence of disease progression in patients with CHB were 9.0\% and 16.2\%, and that of HCC development was 3.6\% and 9.3\%, respectively. In a subgroup of patients with liver cirrhosis, the 3-year and 5-year cumulative incidence was increased to 15.5\% and 30.2\%, respectively, for disease progression and 9.4\% and 21.9\%, respectively, for HCC development.

Several clinical factors have been associated with disease progression and development of HCC during long-term oral NA therapy in CHB patients. A Korean study involving 240 patients with HBV-related liver cirrhosis receiving oral NAs reported that hepatic decompensation was correlated with serum level of HBV DNA at the last follow-up, development of HCC during followup, platelet count, prothrombin time, Child-Pugh score, and the presence of diabetes mellitus, whereas age was the only independent factor correlated with the development of HCC. ${ }^{29}$ Consistent with these observations, the present study showed that age $>50$ years and cirrhosis were significant factors for disease progression and age $>50$ years, family history of HCC, and cirrhosis were significant factors for HCC development. Given the previous studies, advanced age, male gender, high viral load, cirrhosis is well established risk factors for HCC. Thus, patients who had a risk of HCC should be recommended to undergo regular surveillance for HCC even under oral NA therapy. In the era of oral NAs, virological response is considered to be an important factor associated with prognosis of CHB. Suboptimal viral suppression (HBV DNA $>10^{5}$ copies $/ \mathrm{mL}$ at last follow-up) has been implicated as an important risk factor of death and hepatic decompensation and was linked to an increased risk of HCC. ${ }^{29}$ A systematic review of studies in $\mathrm{CHB}$ patients receiving NA therapy showed that HBeAg negative status at baseline and failure to remain in virological remission were associated with an increased risk of HCC. ${ }^{30}$ A recent study involving ETV treated patients with cirrhosis demonstrated that maintained viral suppression reduces the risk of hepatic events, HCC, liver-related mortality, and all-cause mortality after adjustment for model for end-stage liver disease score. ${ }^{27}$ Interestingly, in our study, longer duration (>12 months) of maintained virological response significantly reduced the risks of disease progression (HR, 0.19; 95\% CI, 0.09 to 0.37) and HCC development (HR, 0.09; 95\% CI, 0.04 to 0.21 ). Meanwhile, our study showed that 21 patients (6.4\%) among 330 patients with CHB progressed to cirrhosis during antiviral therapy. Older age, diabetes, lower platelet count, no maintained virological response ( $>12$ months) were associated with progression to cirrhosis from CHB. In addition, initial treatment with ETV was associated with achievement of maintained virological response ( $>12$ months). These findings imply that antiviral therapy should be started as soon as possible before progression to cirrhosis or aging. To achieve and to maintain virological response, potent antiviral agents seem to be important.

Our study had several limitations. First, we did not compare the cumulative incidence of disease progression or HCC development between oral NA treated patients and untreated patients. Second, the retrospective design of study might affect the certain clinical data collection. There was higher percentage of cirrhosis patients and small sample size in the ETV treated group than in the other NAs treated group. Thus we cannot conclude the difference of risk for disease progression or HCC between ETV treated patients and other NAs treated patients.

In conclusion, the cumulative incidence of disease progression and HCC development in CHB patients under long-term oral NA therapy may increase according to the treatment duration. Baseline factors including age $>50$ years, pre-existing cirrhosis, family history of HCC, and duration of maintained virological response to NA therapy were significantly associated with disease progression or HCC development. Longer duration of maintained virological response $>12$ months significantly lowered the risk of disease progression and HCC development in CHB patients under long-term oral NA therapy.

\section{CONFLICTS OF INTEREST}

No potential conflict of interest relevant to this article was reported.

\section{ACKNOWLEDGEMENTS}

This study was supported by research funds of the Chonbuk National University in 2014.

\section{REFERENCES}

1. Lavanchy D. Hepatitis B virus epidemiology, disease burden, treat- 
ment, and current and emerging prevention and control measures. J Viral Hepat 2004;11:97-107.

2. Chen CJ, Yang HI, Su J, et al. Risk of hepatocellular carcinoma across a biological gradient of serum hepatitis B virus DNA level. JAMA 2006;295:65-73.

3. Iloeje UH, Yang HI, Su J, et al. Predicting cirrhosis risk based on the level of circulating hepatitis B viral load. Gastroenterology 2006;130:678-686.

4. Iloeje UH, Yang HI, Jen CL, et al. Risk and predictors of mortality associated with chronic hepatitis B infection. Clin Gastroenterol Hepatol 2007;5:921-931.

5. Yuen MF, Seto WK, Chow DH, et al. Long-term lamivudine therapy reduces the risk of long-term complications of chronic hepatitis B infection even in patients without advanced disease. Antivir Ther 2007;12:1295-1303.

6. Liaw YF, Sung JJ, Chow WC, et al. Lamivudine for patients with chronic hepatitis B and advanced liver disease. N Engl J Med 2004;351:1521-1531.

7. Di Marco V, Marzano A, Lampertico P, et al. Clinical outcome of HBeAg-negative chronic hepatitis B in relation to virological response to lamivudine. Hepatology 2004;40:883-891.

8. Liaw YF, Gane E, Leung N, et al. 2-Year GLOBE trial results: telbivudine is superior to lamivudine in patients with chronic hepatitis B. Gastroenterology 2009;136:486-495.

9. Yoon EL, Yim HJ, Lee HJ, et al. Comparison of clevudine and entecavir for treatment-naive patients with chronic hepatitis B virus infection: two-year follow-up data. J Clin Gastroenterol 2011;45:893-899.

10. Marcellin P, Chang TT, Lim SG, et al. Adefovir dipivoxil for the treatment of hepatitis B e antigen-positive chronic hepatitis B. N Engl J Med 2003;348:808-816.

11. Hadziyannis SJ, Tassopoulos NC, Heathcote EJ, et al. Adefovir dipivoxil for the treatment of hepatitis B e antigen-negative chronic hepatitis B. N Engl J Med 2003;348:800-807.

12. Schiff ER, Lai CL, Hadziyannis S, et al. Adefovir dipivoxil therapy for lamivudine-resistant hepatitis B in pre- and post-liver transplantation patients. Hepatology 2003;38:1419-1427.

13. Rapti I, Dimou E, Mitsoula P, Hadziyannis SJ. Adding-on versus switching-to adefovir therapy in lamivudine-resistant HBeAgnegative chronic hepatitis B. Hepatology 2007;45:307-313.

14. Lampertico P, Viganò M, Manenti E, Iavarone M, Sablon E, Colombo M. Low resistance to adefovir combined with lamivudine: a 3-year study of 145 lamivudine-resistant hepatitis B patients. Gastroenterology 2007;133:1445-1451.

15. Sherman M, Yurdaydin C, Simsek H, et al. Entecavir therapy for lamivudine-refractory chronic hepatitis B: improved virologic, biochemical, and serology outcomes through 96 weeks. Hepatology 2008;48:99-108.

16. Chang TT, Gish RG, de Man R, et al. A comparison of entecavir and lamivudine for HBeAg-positive chronic hepatitis B. N Engl J Med 2006;354:1001-1010.

17. Lai CL, Shouval D, Lok AS, et al. Entecavir versus lamivudine for patients with HBeAg-negative chronic hepatitis B. N Engl J Med 2006;354:1011-1020.

18. Marcellin P, Heathcote EJ, Buti M, et al. Tenofovir disoproxil fumarate versus adefovir dipivoxil for chronic hepatitis B. N Engl J Med 2008;359:2442-2455.

19. Pawlotsky JM, Dusheiko G, Hatzakis A, et al. Virologic monitoring of hepatitis B virus therapy in clinical trials and practice: recommendations for a standardized approach. Gastroenterology 2008;134:405-415.

20. Han KH, Hong SP, Choi SH, et al. Comparison of multiplex restriction fragment mass polymorphism and sequencing analyses for detecting entecavir resistance in chronic hepatitis B. Antivir Ther 2011;16:77-87.

21. Suk KT, Baik SK, Yoon JH, et al. Revision and update on clinical practice guideline for liver cirrhosis. Korean J Hepatol 2012;18:121.

22. European Association for the Study of the Liver; European Organisation for Research and Treatment of Cancer. EASL-EORTC clinical practice guidelines: management of hepatocellular carcinoma. J Hepatol 2012;56:908-943.

23. Korean Association for the Study of the Liver. KASL clinical practice guidelines: management of chronic hepatitis B. Clin Mol Hepatol 2012;18:109-162.

24. Dienstag JL, Goldin RD, Heathcote EJ, et al. Histological outcome during long-term lamivudine therapy. Gastroenterology 2003;124:105-117.

25. Hadziyannis SJ, Tassopoulos NC, Heathcote EJ, et al. Long-term therapy with adefovir dipivoxil for HBeAg-negative chronic hepatitis B for up to 5 years. Gastroenterology 2006;131:1743-1751.

26. Toyama $\mathrm{T}$, Ishida $\mathrm{H}$, Ishibashi $\mathrm{H}$, et al. Long-term outcomes of add-on adefovir dipivoxil therapy to ongoing lamivudine in patients with lamivudine-resistant chronic hepatitis B. Hepatol Res 2012;42:1168-1174.

27. Wong GL, Chan HL, Mak CW, et al. Entecavir treatment reduces hepatic events and deaths in chronic hepatitis B patients with liver cirrhosis. Hepatology 2013;58:1537-1547.

28. Hosaka T, Suzuki F, Kobayashi M, et al. Long-term entecavir treatment reduces hepatocellular carcinoma incidence in patients with hepatitis B virus infection. Hepatology 2013;58:98-107.

29. Kim CH, Um SH, Seo YS, et al. Prognosis of hepatitis B-related liver cirrhosis in the era of oral nucleos(t)ide analog antiviral agents. J Gastroenterol Hepatol 2012;27:1589-1595.

30. Papatheodoridis GV, Lampertico P, Manolakopoulos S, Lok A. Incidence of hepatocellular carcinoma in chronic hepatitis B patients receiving nucleos(t)ide therapy: a systematic review. J Hepatol 2010;53:348-356. 